\title{
Image-based automatic counting of spotted fever-carrying tick larvae in vitro
}

\author{
Weslley Kelson Ribeiro Figueredo \\ Núcleo de Computação Aplicada \\ Universidade Federal do Maranhão \\ São Luis, Brasil \\ weslley.kelson@nca.ufma.br
}

\author{
Isabella Chaves Sousa \\ Laboratório de Controle de Parasitos \\ Universidade Federal do Maranhão \\ São Luis, Brasil \\ isabellachavess@hotmail.com
}

\author{
João Otávio Bandeira Diniz \\ Núcleo de Computação Aplicada \\ Universidade Federal do Maranhão \\ São Luis, Brasil \\ joao.bandeira@nca.ufma.br
}

\author{
Aristófanes Corrêa Silva \\ Núcleo de Computação Aplicada \\ Universidade Federal do Maranhão \\ São Luis, Brasil \\ ari@nca.ufma.br
}

\author{
Anselmo Cardoso de Paiva \\ Núcleo de Computação Aplicada \\ Universidade Federal do Maranhão \\ São Luis, Brasil \\ paiva@nca.ufma.br
}

\author{
Livio Martins Costa-Junior \\ Laboratório de Controle de Parasitos \\ Universidade Federal do Maranhão \\ São Luis, Brasil \\ livio.martins@ufma.br
}

\begin{abstract}
Spotted fever or tick fever, a disease transmitted by ticks, has a high case-fatality rate when a treatment is not started early. To reduce the cases of tick fever, many acaricides are used for keeping control of tick population. However, these tests are done manually. To choose the most effective acaricide, a specialist has to count how many ticks are dead and how many are still alive after subjecting them to it. This test is time-consuming, so this work aims to automate this procedure. Therefore, it is proposed a computational methodology to automate this procedure through image processing. Through the proposed method, 99,14\% of ticks larvae were found, and $99.34 \%$ of alive and $96.89 \%$ of dead ticks larvae were counted correctly in this work.
\end{abstract}

Keywords-Ticks, Automatic counting, Acaricides, Image processing, FRST

\section{INTRODUÇÃO}

A febre maculosa é uma patologia infecciosa grave transmitida por carrapatos. Apesar da doença possuir cura, o início imediato de tratamento é necessário, caso contrário, a doença manifesta-se de forma severa podendo ter consequências tais como insuficiência respiratória, inflamações no cérebro e paralisia. Esta enfermidade é causada por uma espiroqueta, um filo das bactérias, a Rickettsia rickettsii e é transmitida pela saliva do carrapato. Ao entrar em contato com o organismo humano, a bactéria dissemina-se através dos vasos linfáticos e pequenos vasos sanguíneos, alcançando vários órgãos como fígado, coração e cérebro [1] [2].

Entre 2017 e o primeiro semestre de 2018, 250 casos de febre maculosa foram registrados no Brasil, com 81 mortes [3]. Os casos ocorrem em sua maioria na região Sudeste do país e em regiões rurais, todavia nos últimos anos notou-se uma urbanização da doença. A manifestação da doença dá-se usualmente entre os meses de junho e outubro [1].

No Brasil, o principal vetor é o carrapato-estrela ( $\mathrm{Am}$ blyomma cajennene). Entretanto, os carrapatos dos bovinos,

Agradecimento à Fundação de Amparo à Pesquisa e ao Desenvolvimento Científico e Tecnológico do Maranhão - FAPEMA o Rhipicephalus (Boophilus) microplus, e o carrapatos dos cães (Rhipicephalus sanguineus), podem ser utilizados como modelo de estudos para desenvolvimento de novas técnicas de avaliação da eficácia de carrapaticidas, bem como novas formas de controle. Especialistas na área testam vários acaricidas, substâncias capazes de eliminar carrapatos, em larvas destes tendo em vista analisar a eficácia destes produtos. As larvas destes animais são utilizadas neste teste devido a praticidade de manter-se uma grande quantidade destes indivíduos, permitindo uma melhor representatividade da realidade. A contagem de quantos carrapatos morreram e quantos sobreviveram após 24 horas do tratamento com o produto permite a análise do produto.

Até o momento, trabalhos que buscam a contagem automática ou similares são escassos na literatura. Não é possível encontrar metodologias computacionais que fazem a detecção e contagem automática de carrapatos como maneira de prevenção a doenças. Não obstante, um trabalho [4] utilizou imagens termográficas para contagem de carrapatos e moscados-chifres, mesmo com um domínio de imagem diferente do utilizado neste trabalho, este foi considerado como um trabalho relacionado. Este trabalho utilizou uma base de imagens composta por imagens termográficas adquiridas do gado hospedeiro, entretanto, a contagem desenvolvida é feita através de um software livre chamado ImageJ ${ }^{\circledR}{ }^{1}$. Mesmo que o trabalho desenvolvido seja de contagem automática de carrapatos, [4] avaliaram somente o número de carrapatos sobre o hospedeiro, não contando quantos carrapatos estão vivos e mortos, além de imagens adquiridas em condições diferentes.

Sendo assim, o objetivo desejado é propor um método completamente automático de contagem de carrapatos, que seja capaz de auxiliar os especialistas na tarefa de verificação da eficácia no tratamento contra a proliferação de carrapatos

\footnotetext{
${ }^{1}$ https://imagej.nih.gov/ij/
} 
e, consequentemente, visando a redução na ocorrência de mais casos de febre maculosa.

Com o intuito de automatizar o processo de contagem, propõe-se aplicar técnicas de processamento de imagem sobre as imagens adquiridas pelos especialistas responsáveis pela contagem de larvas de carrapato. o método proposto faz uso de uma técnica de compensação de iluminação para segmentação do carrapato. Para contagem e rastreamento das larvas, utilizase uma técnica de detecção de círculos na imagem, o fast radial symmetry transform (FRST) [5] e uma técnica de acompanhamento de deslocamento de partículas baseado no trabalho desenvolvido por [6].

O trabalho propõe um método completamente automático para auxiliar especialista na contagem de carrapatos para verificar a eficácia de acaricidas no controle da população de carrapatos, e consequentemente, auxiliar na diminuição de casos da febre maculosa. Pode-se listar uma série de contribuições que o trabalho englobou: (a) um método de segmentação/detecção de larvas de carrapatos automática baseada na compensação de iluminação e operações morfológicas; (b) utilização de uma técnica de detecção de regiões com formato elíptico para encontrar larvas em vídeos; e (c) contagem automática de larvas vivas e mortas. A partir disto, o método proposto pode ser utilizada como parte do processo no controle da população de carrapatos.

Este trabalho possui 4 seções. Na Seção 2, serão apresentados os materiais e métodos utilizados. A seguir, na Seção 3, serão discutidos os resultados atingidos pela método proposta. Na Seção 4, é feita uma conclusão do trabalho, na qual fazse uma avaliação do trabalho e propõe-se novas abordagens como trabalhos futuros.

\section{MATERIAIS E MÉTODOS}

Os materiais e métodos aplicados no trabalho são apresentados nesta seção. O método proposto está dividido em três fases. Na primeira fase, é feita a aquisição de imagens. $\mathrm{Na}$ segunda, é realizada a segmentação das larvas. A última fase consiste no rastreamento da movimentação das larvas. Se alguma larva apresentar movimentação no vídeo, esta é considerada viva, caso contrário, morta.

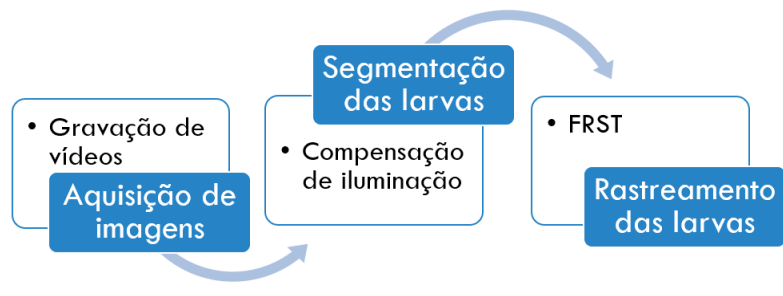

Fig. 1. Pipeline da metodologia proposta

\section{A. Aquisição de imagens}

A base de imagens é composta por vídeos de 3 amostras de larvas diferentes, cada amostra foi gravada duas vezes em tempos distintos, gerando 6 vídeos. Nos 6 vídeos da base, pode-se observar uma superfície, duas folhas de papel, na qual as larvas de carrapatos estavam armazenadas, sobre uma mesa. Os vídeos de 30 frames foram gravados a uma distância de 15 centímetros entre o dispositivo utilizado para a aquisição e a superfície. Há uma movimentação inicial do dispositivo no inicio da gravação, causado por ação externa durante o posicionamento do dispositivo, portanto, os primeiros 5 segundos dos vídeos são ignorados. Desconsiderando esse tempo inicial, são colhidos 100 frames de cada vídeo para a montagem da base. Na Figura 2, mostra-se um dos frames retirado de um dos vídeo. Os vídeos adquiridos foram gravados nas mesmas condições em que são feitas as contagens manuais pelos especialistas. A contagem manual do especialista é feita ao término da gravação, pois as larvas são armazenadas em outro local durante o processo de contagem.

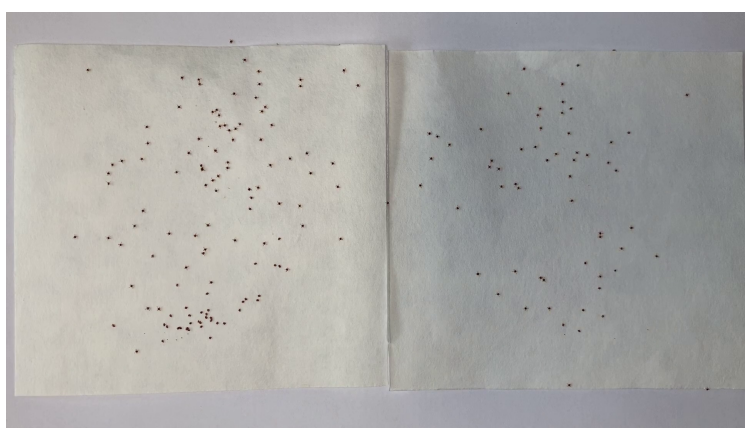

Fig. 2. Frame do vídeo

\section{B. Segmentação das larvas}

Nesta seção, é exposta a técnica utilizada para a segmentação no método proposto. A técnica desenvolvida é a compensação de iluminação, que busca melhorar a imagem aumentando o contraste entre o fundo e objeto a ser detectado [7].

Para detectar as de regiões de interesse, utilizou-se uma técnica automática baseado na compensação de iluminação composta por uma sequência de processamentos de imagem que buscam destacar os carrapatos em relação ao fundo. Esta técnica mostrou-se mais adequada devido a variação de iluminação nas imagens adquiridas, visando destacar as larvas em relação ao fundo.

A técnica aplicado nesta etapa tem como base a implementação de compensação de iluminação para imagens de texto desenvolvida por [7]. Este consiste em 5 etapas: melhoria do contraste, detecção das bordas, localização do texto, distribuição da iluminação e balanceamento da luz (Figura 3).

A primeira etapa consiste em melhorar o contraste entre o carrapato e o fundo da imagem. Essa melhoria é feita através da Equação 1, onde $h r$ é o valor de pixel com quantidade de pixels maior que a raiz quadrada da quantidade de pixels, I é a imagem, pvj é o valor de pixel $j$, onde $I$ é uma constante para redução de brilho de valor 0.4 , e as demais variáveis são utilizados como fatores para melhoria do contraste, com valores definidos por [7]. 


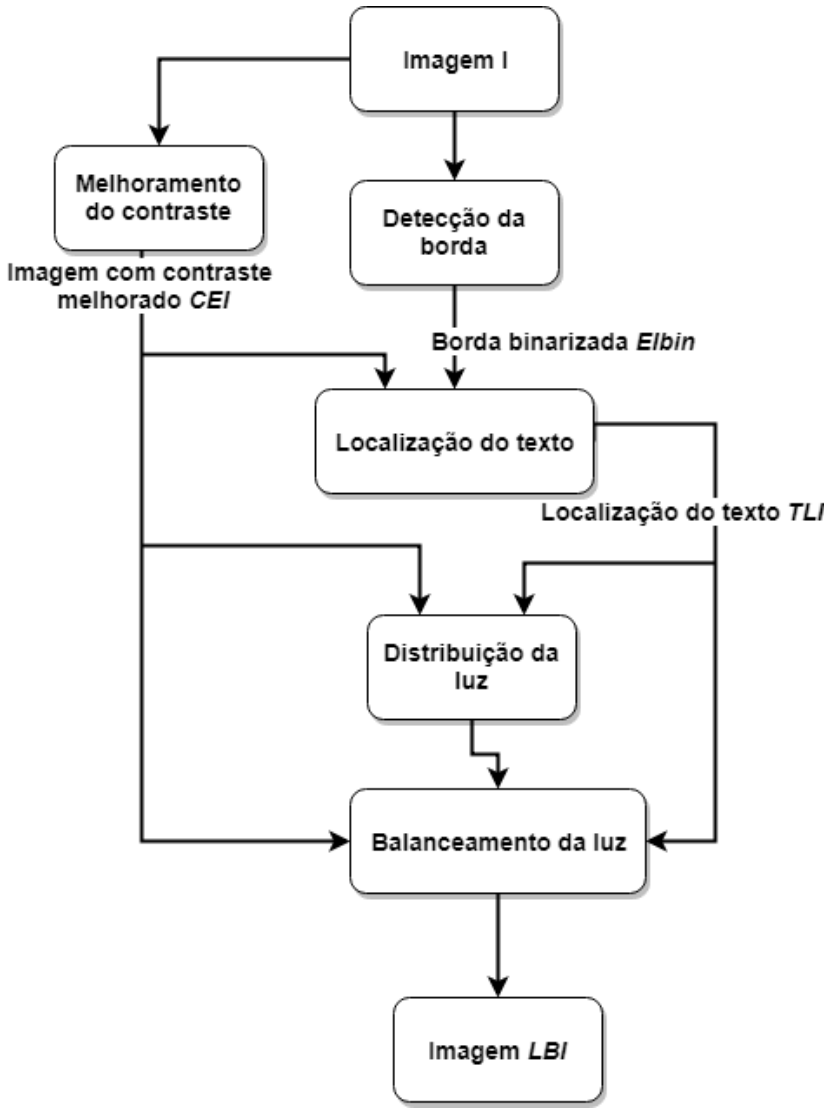

Fig. 3. Pipeline da técnica de compensação de iluminação Fonte: baseado em [7]

$$
C E I\left(p v_{j}\right)=\left(I\left(p v_{j}\right)-(h r+50 * c)\right) * 2
$$

A etapa de detecção de borda consiste na criação de 4 máscaras de tamanho $3 \times 3$ em quatro orientações, mostradas na Figura 4, que são aplicadas sobre a imagem original em tons de cinza. Posteriormente, é gerada uma imagem com a média dos valores das quatro máscaras, que é limiarizada $\left(E I_{b i n}\right)$. Ao final, a imagem com contraste melhorado também é limiarizada $\left(C E I_{b i n}\right)$.

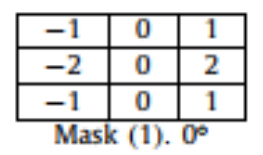

\begin{tabular}{|c|c|c|}
\hline-1 & -2 & -1 \\
\hline 0 & 0 & 0 \\
\hline 1 & 2 & 1 \\
\hline
\end{tabular}

Mask (3). $90^{\circ}$

\begin{tabular}{|c|c|c|}
\hline-2 & -1 & 0 \\
\hline-1 & 0 & 1 \\
\hline 0 & 1 & 2 \\
\hline \multicolumn{4}{|c|}{ Mask (2). $45^{\circ}$}
\end{tabular}

\begin{tabular}{|c|c|c|}
\hline 0 & 1 & 2 \\
\hline-1 & 0 & 1 \\
\hline-2 & -1 & 0 \\
\hline
\end{tabular}

Mask (4). $135^{\circ}$
Fig. 4. Máscaras para detecção de bordas

Para localizar das larvas (TLI), ambas as imagens resultantes da etapa anterior são combinadas. Onde $T L I\left(p v_{j}\right)$ é igual a 255 , se $E I_{b i n}\left(p v_{j}\right)$ e $C E I_{b i n}\left(p v_{j}\right)$ forem igual a 255 , e 0 , caso contrário, e onde $E I_{b i n}$ e $C E I_{b i n}$ são a média e a imagem com contraste melhorado limiarizada, respectivamente. Enfim, é feita uma erosão sobre a imagem $T L I$.

A distribuição da iluminação $L D I$ é feita em $T L I$, onde a imagem é percorrida coluna a coluna, quando um pixel for igual a 0 , este é considerado larva. O primeiro pixel encontrado na coluna é chamado head $(h)$ e o último end $(e)$. Em seguida, é aplicada a Equação 2, onde $n$ é número de pixels em uma área de texto (larvas em nosso trabalho), definida pela busca, e $m$ é um pixel que varia de 0 a $n . m v p_{h}$ e $m p v_{e}$ são definidas nas Equações 3 e 4 , onde $\mathrm{k}$ varia de 0 a 4 . Posteriormente, aplica-se um filtro da média sobre o resultado, gerando a imagem $L D I$.

$$
\begin{gathered}
D L I\left(p v_{h+m}\right)=C E I\left(p v_{h-1}\right)+f \frac{m p v_{e}-m p v_{h}}{n} *(m+1) \\
m p v_{h}=M A X\left(C E I\left(p v_{h-k}\right)\right) \\
m p v_{e}=M A X\left(C E I\left(p v_{e+k}\right)\right)
\end{gathered}
$$

O balanceamento da luz $(L B I)$ é feito através do resultado de $T L I$ (Equação 5). Onde $b l$ é um parâmetro com valor, definido pelo autor, de 260. Com esta operação, encontra-se o resultado da etapa de geração de marcação, como é mostrado na Figura 5, onde é feita uma comparação entre a imagem original (Figura 5(a)) e o resultado do processamento (Figura $5(\mathrm{a})$ ).

$$
L B I\left(p v_{j}\right)= \begin{cases}\frac{b l}{L D I\left(p v_{j}\right)} *\left(C E I\left(p v_{j}\right)\right), & \text { se } T L I=0 . \\ \frac{b l * 1.5}{L D I\left(p v_{j}\right)} *\left(C E I\left(p v_{j}\right)\right), & \text { caso contrário. }\end{cases}
$$

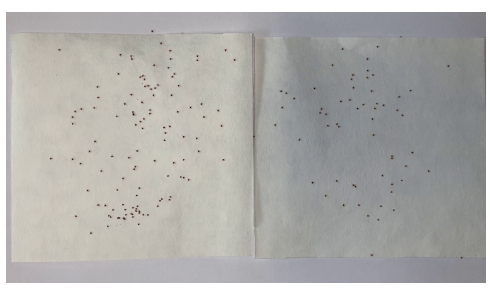

(a)

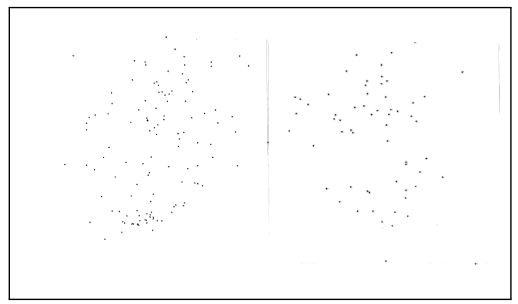

(b)

Fig. 5. Compensação da iluminação: (a) frame original e (b) resultado da compensação

Para iniciar o processo de contagem de larvas de larvas vivas e mortas, primeiramente é preciso segmentar cada carrapato 
e, em seguida, verificar a movimentação de cada larva entre os frames.

Nesta etapa, apresenta-se a etapa final de segmentação das larvas. Como pode-se observar na Figura 5, ainda há ruído na imagem que pode impossibilitar a contagem correta dos parasitas. Para esta tarefa, aplicou-se uma dilatação de formas elípticas na imagem para dar destaque as larvas. Aplicouse o OTSU [8] para a limiarização, eliminando ruído de baixa intensidade de tom de cinza. Ao identificar os contornos restantes na imagem, consegue-se extrair contornos com áreas muito pequenas e muito grandes, que não podem ser classificados como larvas.

\section{Rastreamento da movimentação}

Para acompanhar o movimento, quando há, dos carrapatos ao longo dos frames e descobrir quais estão mortos ou vivos, é feita uma etapa de rastreamento da larva ao longo do vídeo. Inicia-se esta etapa detectando-se objetos de interesse com forma elíptica na imagem. Logo após, faz-se o rastreamento baseando-se em dois frames: o frame anterior $(t-1)$ e frame atual $(t)$.

Para este rastreamento ser feito, primeiramente precisa-se encontrar a posição inicial das larvas, e acompanhar a posição de cada larva nos frames seguintes. Para atingir esta finalidade, utilizou-se o algoritmo de fast radial symmetry transform (FRST).

A técnica FRST é uma transformada que infere centros de simetria radial. Usada para identificar pontos que possuem aproximadamente um raio específico $(n)$. Esse raio é definido de acordo com o raio aproximado do objeto a ser encontrado. Por possuem forma elíptica, as larvas podem ser detectadas através do FRST.

O FRST consiste em detectar se o pixel é positivamente $(P+v e(P))$ ou negativamente $(P-v e(P)$ afetado pelo gradiente. Sendo que isto é calculado através das Equações 6 e 7. Onde $g(p)$ é o vetor gradiente para o qual o pixel está apontando.

$$
\begin{aligned}
& (P+v e(P))=p+\operatorname{round}\left(\frac{g(p)}{\|g(p)\|} n\right) \\
& (P-\operatorname{ve}(P))=p-\operatorname{round}\left(\frac{g(p)}{\|g(p)\|} n\right)
\end{aligned}
$$

Então, a magnitude $(M n)$ e orientação $(O n)$ são calculadas pelas Equações 8, 9, 10 e 11. Após, é aplicada as Equações 12,13, 14 e 15 que origina os objetos detectados pelo FRST, onde $A_{n}$ é uma gaussiana de duas dimensões e $\alpha$ é um parâmetro definido via testes.

$$
\begin{array}{r}
O_{n}(P+v e(P))=O_{n}(P+v e(P))+1 f \\
O_{n}(P-v e(P))=O_{n}(P-v e(P))-1 \\
M_{n}(P+v e(P))=M_{n}(P+v e(P))+\|g(p)\| \\
M_{n}(P-v e(P))=M_{n}(P-v e(P))-\|g(p)\|
\end{array}
$$

$$
\begin{gathered}
S_{n}=F_{n} * A_{n} \\
F_{n}(P)=\left\|O_{n}(P)\right\|^{\alpha} M_{n}(P) \\
O_{n}(P)=\frac{O_{n}}{\max _{p}\left\{\left\|O_{n(P)}\right\|\right\}} \\
M_{n}(P)=\frac{M_{n}}{\max _{p}\left\{\left\|M_{n(P)}\right\|\right\}}
\end{gathered}
$$

As regiões geradas pelo FRST tem tamanhos diferentes e podem sobrepor partes distintas do corpo da larva. A partir destas regiões de interesse, detecta-se cada contorno de cada área, gera-se bounding boxes sobre cada contorno e calculase os centros destes, que serão definido como o marcador de larva. Na Figura 6, apresenta-se parte do frame antes e depois da marcação.

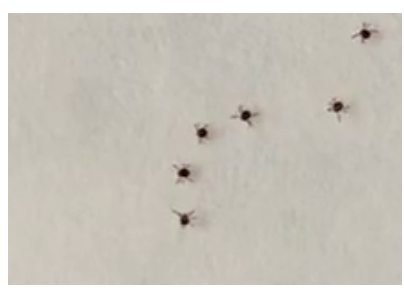

(a)

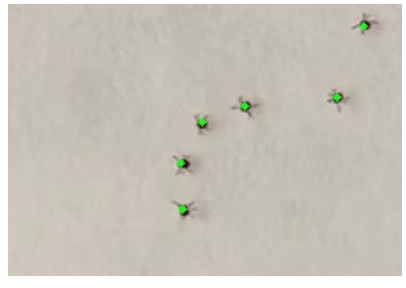

(b)
Fig. 6. Representação da marcação das larvas (a) Larvas sem marcação. (b) Larvas após marcação.

Com as regiões definidas pelo FRST, é viável manter as posições iniciais dos carrapatos detectados no primeiro frame do vídeo salvas. As regiões identificadas no próximo frame ( $t$ ) são comparadas com o frame anterior $(t-1)$ através da distância euclidiana. Um ponto no próximo frame é considerado representante de uma mesma larva se essa distância for a menor possível da mesma larva no frame anterior. Ao término do processo, os pontos do último frame são comparados aos pontos do primeiro, e se houver mudança da posição, a larva é considerada como viva. Também é considerada uma pequena janela de movimentação de 3 pixels, pois um ponto, que marca uma larva, pode identificar outra parte da mesma larva frames posteriores, e também, caso ocorra alguma ação externa que faça com que o dispositivo utilizado se mova, dando o aspecto de movimento em larvas mortas. A movimentação de algumas larvas ao longo do tempo pode ser observada na Figura 7.

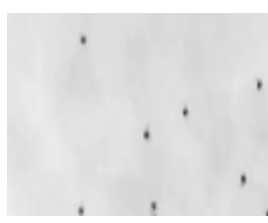

(a)

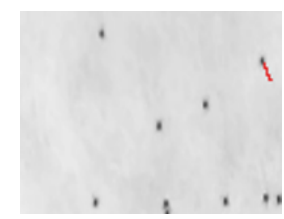

(b)
Fig. 7. Movimentação da larva através do tempo. (a): Larvas no frame 0. (b): Larvas no frame 50. A linha em vermelho mostra a trajetória de uma larva

Com o término desta etapa, consegue-se identificar, a partir da movimentação das larvas através do tempo de vídeo, se 
determinada larva está viva ou morta. Ao testar o acaricida, para cada vídeo o especialista elenca quantas larvas existem em cada vídeo e quantas ficaram vivas ou mortas. Assim, é possível avaliar o quão o método proposto foi robusto, tanto na segmentação quanto na contagem de larvas vivas e mortas.

\section{RESULTADOS}

Serão apresentados e discutidos os resultados obtidos na segmentação e no rastreamento das larvas nesta seção. Estes são exibidos em grupo de vídeos por amostra de larvas utilizada durante a aquisição das imagens.

\section{A. Segmentação das larvas}

A segmentação com a compensação de iluminação foi capaz de identificar áreas como larvas, entretanto, algumas destas áreas também foram originadas devido a sombra e ruído na imagem. Foi possível remover algumas destas áreas, pois não são detectadas como pontos de interesse com raio $n$ pelo FRST. Assim como, em razão da sombra tornar larvas maiores que seu real tamanho, algumas foram perdidas. Todavia, a perda foi mínima e o resultado é bem próximo ao indicado pelo especialista.

Com a segmentação, na primeira amostra, identificou-se 176 áreas de interesse classificadas como larvas de carrapatos no primeiro vídeo e 174 no segundo. E de acordo com a contagem feita manualmente pelo especialista, havia 176 larvas nesta amostra. Na segunda amostra, o método proposto foi capaz de detectar 166 e 167 larvas no primeiro e segundo vídeo respectivamente, de um total de 171 larvas, ou seja mais de $97,07 \%$ das larvas foram detectadas. Por fim, no terceiro amostra, a contagem feita pelo especialista resultou em 120 larvas, esta segmentação permitiu contar 124 larvas no primeiro vídeo e 122 no segundo vídeo. A análise do ambiente e os relatos dos especialistas, sugeriram que algumas larvas podem ser perdidas durante o processo de contagem, por estarem em constante movimento.

Apesar da compensação gerar bons resultados, ainda ocorrem alguns erros devido a sombra e ruídos gerados sobre a superfície. Pontos que não eram carrapatos foram selecionados como tais. Em alguns casos, quando as larvas estavam muito próximas umas das outras, estas eram confundidas como uma única larva. Apesar do FRST inferir pontos na imagem conseguir separar algumas destas, a abordagem não refletiu o mesmo resultado em todas as larvas que estavam nesta configuração, assim, justifica-se algumas larvas foram perdidas. Ainda assim, vale destacar que o método conseguiu alcançar uma alta taxa de acerto perdendo poucas larvas, o que auxiliaria de forma expressiva na contagem visual do especialista.

\section{B. Rastreamento da movimentação}

Nesta etapa, após a segmentação utilizando a compensação de iluminação, feita a contagem das larvas baseando-se na movimentação delas no decorrer dos vídeos. Como é apresentado na Seção II-C, o frame atual do vídeo é comparado ao frame anterior, buscando fazer o acompanhamento de cada movimento da larva. O valor do parâmetro $n$ do FRST foi definido como 2. Como, até então, não foi possível encontrar trabalhos na literatura que desenvolvessem um método similar ao deste trabalho, a comparação com outros trabalhos torna-se inviável. Na Figura 8, pode-se observar o movimento de uma larva específica ao longo do tempo, destacada por um círculo vermelho nos frames após a marcação com o FRST.

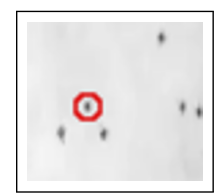

(a)

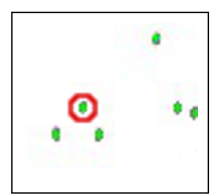

(b)

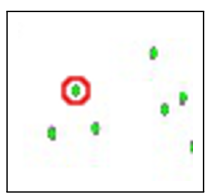

(c)

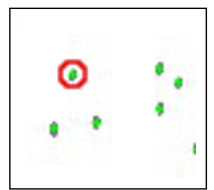

(d)

Fig. 8. Marcação do FRST nas larvas através do tempo. (a) Larvas no frame 0 na imagem original. (b) Larvas no frame 0 (c) Larvas no frame 30 (d) Larvas no frame 60.

No método desenvolvido com a compensação de iluminação, dentre as 176 larvas encontradas na primeira amostra, 124 foram classificadas como vivas e 52 como mortas em $v_{1.1}$, e em $v_{1.2}$ foram contadas 127 vivas e 47 mortas. $\mathrm{Na}$ contagem feita pelo especialista, foram encontrados, também, 176 larvas, sendo que 126 larvas estavam vivas e 50 mortas (Tabela I). Percebe-se um aumento, no número de larvas vivas no vídeo $v_{1.2}$, devido principalmente a classificação de pixel de sombra como larva em movimento em alguns frames na segmentação.

TABLE I

RESULTADO DO MÉTODO NA PRIMEIRA AMOSTRA.

\begin{tabular}{ccccc} 
& Vídeo & Larvas vivas & Larvas mortas & Total \\
\hline Especialista & & 126 & 50 & 176 \\
\hline \multirow{2}{*}{ Método proposto } & $v_{1.1}$ & $\mathbf{1 2 4}$ & $\mathbf{5 2}$ & $\mathbf{1 7 6}$ \\
& $v_{1.2}$ & 127 & 47 & 174 \\
\hline
\end{tabular}

Na segunda amostra, ambos os resultados atingiram valores próximos a contagem do especialista para larvas vivas. Todavia, ao que se refere a quantidade de larvas mortas, o método teve um resultado ligeiramente diferente da contagem real. $\mathrm{O}$ método com a compensação permitiu encontrar 93 larvas vivas e 73 mortas no vídeo $v_{2.1}$, e 94 vivas e 73 mortas em $v_{2.2}$. A Tabela II compara os resultados obtidos. Percebeu-se uma redução dos resultados em relação a quantidade de larvas vivas e mortas, ocorrendo devido a larvas muito próximas entre si.

$\mathrm{Na}$ Tabela III, observa-se que o método obteve um resultado menos balanceado na amostra 3. Ainda que houve uma diferença maior no resultado na quantidade de larvas vivas entre $v_{3.1}$ e $v_{3.2}$ do que nas demais amostra com o mesmo método, este ainda manteve uma quantidade de larvas próximo ao real, acrescentando uma larva no vídeo $v_{3.1}$ e perdendo 
TABLE II

RESUlTAdO DO MÉTOdO NA SEGUNDA AMOSTRA.

\begin{tabular}{ccccc} 
& Vídeos & Larvas vivas & Larvas Mortas & Total \\
\hline Especialista & & 94 & 77 & 171 \\
\hline \multirow{2}{*}{ Método proposto } & $v_{1.1}$ & 93 & 73 & 166 \\
& $v_{1.2}$ & $\mathbf{9 4}$ & $\mathbf{7 3}$ & $\mathbf{1 6 7}$ \\
\hline
\end{tabular}

uma larva morta $v_{3.2}$. O resultado variou nos dois vídeos. A análise deste vídeo indicou que as sombras nas bordas possam ter confundido o método.

TABLE III

RESUlTADO DO MÉTODO NA TERCEIRA AMOSTRA.

\begin{tabular}{ccccc} 
& Vídeos & Larvas vivas & Larvas mortas & Total \\
\hline Especialista & & 86 & 34 & 120 \\
\hline \multirow{2}{*}{ Método proposto } & $v_{1.1}$ & 87 & 37 & 124 \\
& $v_{1.2}$ & $\mathbf{8 9}$ & $\mathbf{3 3}$ & $\mathbf{1 2 2}$ \\
\hline
\end{tabular}

Os melhores resultados para cada amostra, destacados em negrito nas tabelas, mostrou que o método é bem eficaz. Considerando-se os melhores resultados de todas as amostras e desconsiderando os excessos de larvas, conseguiu-se detectar 99,14\% das larvas do total, o método foi capaz de identificar 99,34\% das larvas vivas corretamente, e 96,89\% das larvas mortas.

\section{CONCLUSÃo E TRABALHOS FUturos}

A automatização do processo de contagem automática de larvas in vitro gerará resultados de testes com acaricidas de forma rápida, permitindo que uma maior quantidade de produtos sejam testados e de obter-se uma decisão mais rápida e eficaz de qual acaricida deve ser usado, impedindo a proliferação destas larvas e, por consequência, prevenir a disseminação de febre maculosa. Os resultados mostraram-se robustos, apesar da perda de algumas larvas, a contagem do método foi bem próxima da contagem manual do especialista, permitindo que este analise a amostra de forma mais rápida.

Para atingir melhores resultados, novas técnicas de segmentação visando evitar perdas e excesso serão investigadas. E, serão pesquisados novos meios de detecção das larvas durante o rastreamento, como a probabilidade de uma larva manter-se na trajetória iniciada, impedindo que larvas sejam classificadas erroneamente quando há a colisão entre larvas. Assim como fazer a aquisição de novas imagens para a base através da gravação de novos vídeos.

Os resultados alcançados até o momento apontam que o método proposto é bastante promissor. Assim este pode tornarse uma ferramenta de auxílio aos especialistas nesta tarefa exaustiva que é a contagem manual das larvas. O método ainda está em desenvolvimento e almeja melhores resultados, empenhando-se em evitar principalmente perdas de larvas na contagem.

\section{REFERÊNCIAS}

[1] F. d. S. D. Fiol, F. M. Junqueira, M. C. P. d. Rocha, M. I. d. Toledo, and S. Barberato Filho, "A febre maculosa no brasil," Revista Panamericana de Salud Pública, vol. 27, pp. 461-466, 2010.
[2] R. P. de Araújo, M. B. M. de Albuquerque Navarro, and T. A. de Oliveira Cardoso, "Febre maculosa no brasil: estudo da mortalidade para a vigilância epidemiológica." Cadernos Saúde Coletiva, vol. 24, no. 3, 2016.

[3] G1, "Em 18 meses, país registra 250 casos de febre maculosa com 81 mortes," 2018, acessado em 20 de janeiro de 2019. [Online]. Available: https://g1.globo.com/jornal-nacional/noticia/2018/08/02/em-18meses-pais-registra-250-casos-de-febre-maculosa-com-81-mortes.ghtml

[4] P. D. Cortivo, E. Dias, J. O. J. Barcellos, V. Peripolli, J. B. G. Costa Jr, B. S. L. Dallago, and C. M. McManus, "Use of thermographic images to detect external parasite load in cattle," Computers and Electronics in Agriculture, vol. 127, pp. 413-417, 2016.

[5] G. Loy and A. Zelinsky, "A fast radial symmetry transform for detecting points of interest," in European Conference on Computer Vision. Springer, 2002, pp. 358-368.

[6] S. Aghayee, D. E. Winkowski, Z. Bowen, E. E. Marshall, M. J. Harrington, P. O. Kanold, and W. Losert, "Particle tracking facilitates real time capable motion correction in $2 \mathrm{~d}$ or $3 \mathrm{~d}$ two-photon imaging of neuronal activity," Frontiers in neural circuits, vol. 11, p. 56, 2017.

[7] K.-N. Chen, C.-H. Chen, and C.-C. Chang, "Efficient illumination compensation techniques for text images," Digital Signal Processing, vol. 22, no. 5, pp. 726-733, 2012.

[8] N. Otsu, "A threshold selection method from gray-level histograms," IEEE transactions on systems, man, and cybernetics, vol. 9, no. 1, pp. 62-66, 1979. 